\title{
AN EXTENSION OF THE TOPOLOGICAL DEGREE IN HILBERT SPACE
}

\author{
J. BERKOVITS AND C. FABRY
}

Received 16 June 2004

We define classes of mappings of monotone type with respect to a given direct sum decomposition of the underlying Hilbert space $H$. The new classes are extensions of classes of mappings of monotone type familiar in the study of partial differential equations, for example, the class $\left(S_{+}\right)$and the class of pseudomonotone mappings. We then construct an extension of the Leray-Schauder degree for mappings involving the above classes. As shown by (semi-abstract) examples, this extension of the degree should be useful in the study of semilinear equations, when the linear part has an infinite-dimensional kernel.

\section{Introduction}

We will introduce classes of mappings of monotone type with respect to a given projection $P: H \rightarrow E$, where $E$ is a closed linear subspace of a real Hilbert space $H$. We give a systematic classification and show how the new classes are related to each other, how they are related to the corresponding traditional classes and how they stand perturbations. The main result of this note is the construction of a topological degree for mappings of the form

$$
F=Q(I-C)+P N: \bar{G} \longrightarrow H
$$

where $G$ is an open bounded set in $H, C$ is compact, $N$ is a bounded demicontinuous mapping satisfying a generalized condition $\left(S_{+}\right)$with respect to $P$, and $Q=I-P$. The degree defined in this note is a unique extension of the Leray-Schauder degree. It is single-valued and has the usual properties of degree, such as additivity of domains and invariance under homotopies. The present construction extends and completes the results obtained in [3].

Some classes of mappings we consider here have appeared earlier in the literature. For instance, Mawhin and Willem $[11,12,13,14]$ have introduced similar monotonicity conditions in the study of semilinear wave equations, using Galerkin's method together with Leray-Schauder and coincidence degrees. In the present note, we concentrate on the definition of a degree for mappings involving functions satisfying the monotonicity 
conditions; we will not pursue for concrete applications, but will give indications on the kind of context in which the degree theory could be effectively used.

The paper is organized as follows. In Section 2, we introduce classes of mappings of monotone type with respect to any bounded linear operator $T: H \rightarrow H$. We will mainly deal with the special choice $T=P$, an orthogonal projection onto a closed linear subspace $E$. We will give the main properties of the new classes of mappings of monotone type: $\left(S_{+}\right)_{T}, T$-pseudomonotone, $T$-quasimonotone and $(M)_{T}$. We show their relations to each other and to the traditional classes as well. Most of results are direct consequences of the definitions, but are included here for the sake of completeness. In Section 3, we will construct a topological degree for a class of mappings related to the composition $E \oplus E^{\perp}$ of $H$. The degree is a unique extension of the Leray-Schauder degree in Hilbert space. The construction completes the extension of degree theory given in [3], (see also [4]). Section 4 is devoted to the basic properties and to the uniqueness of the degree. In Section 5, we consider, on a general level, the use of the topological degree to obtain existence results. In Section 6, we will consider systems of semilinear equations, which motivate our definition of degree. It allows indeed to limit the monotonicity hypothesis to some components only of the system. We close this note by a short discussion of semilinear equations with non-symmetric linear part. It turns out that in certain cases we can apply the degree theory, provided the nonlinearity is of class $\left(S_{+}\right)_{T}$ with respect to a suitable operator $T$.

\section{On the mappings of monotone type}

Throughout, $H$ will denote a real separable Hilbert space, with inner product $\langle\cdot, \cdot\rangle$ and corresponding norm $\|\cdot\|$. We recall some basic definitions. A mapping $F: H \rightarrow H$ is

(i) bounded, if it takes any bounded set into a bounded set;

(ii) demicontinuous, if $u_{j} \rightarrow u$ (norm convergence) implies $F\left(u_{j}\right) \rightarrow F(u)$ (weak convergence);

(iii) compact, if it is continuous and the image of any bounded set is relatively compact;

(iv) Leray-Schauder type, if it is of the form $I-C$, where $C$ is compact;

Let $T: H \rightarrow H$ be a bounded linear operator. We will define classes of mappings of monotone type with respect to $T$. A mapping $F: H \rightarrow H$ is said

(i) $T$-monotone, if $\langle F(u)-F(v), T(u-v)\rangle \geq 0$ for all $u, v \in H$;

(ii) of class $\left(S_{+}\right)_{T}$, if for any sequence $\left(u_{j}\right), u_{j}=v_{j}+z_{j}, v_{j} \in \operatorname{Ker} T, z_{j} \in(\operatorname{Ker} T)^{\perp}$ with $u_{j} \rightarrow u$ and $v_{j} \rightarrow v$ such that $\lim \sup \left\langle F\left(u_{j}\right), T\left(u_{j}-u\right)\right\rangle \leq 0$, it follows that $u_{j} \rightarrow u$;

(iii) $T$-pseudomonotone $\left(F \in(P M)_{T}\right)$, if for any sequence $\left(u_{j}\right), u_{j}=v_{j}+z_{j}, v_{j} \in \operatorname{Ker} T$, $z_{j} \in(\operatorname{Ker} T)^{\perp}$ with $u_{j} \rightarrow u$ and $v_{j} \rightarrow v$ such that $\lim \sup \left\langle F\left(u_{j}\right), T\left(u_{j}-u\right)\right\rangle \leq 0$, it follows that $F\left(u_{j}\right) \rightarrow F(u)$ and $\left\langle F\left(u_{j}\right), T\left(u_{j}-u\right)\right\rangle \rightarrow 0$;

(iv) $T$-quasimonotone $\left(F \in(Q M)_{T}\right)$, if for any sequence $\left(u_{j}\right), u_{j}=v_{j}+z_{j}, v_{j} \in \operatorname{Ker} T$, $z_{j} \in(\operatorname{Ker} T)^{\perp}$ with $u_{j} \rightarrow u$ and $v_{j} \rightarrow v$ such that $\limsup \left\langle F\left(u_{j}\right), T\left(u_{j}-u\right)\right\rangle \leq 0$, it follows that $\left\langle F\left(u_{j}\right), T\left(u_{j}-u\right)\right\rangle \rightarrow 0$;

(v) of class $(M)_{T}$, if for any sequence $\left(u_{j}\right), u_{j}=v_{j}+z_{j}, v_{j} \in \operatorname{Ker} T, z_{j} \in(\operatorname{Ker} T)^{\perp}$ with $u_{j} \rightarrow u$ and $v_{j} \rightarrow v$ such that $\lim \sup \left\langle F\left(u_{j}\right), T\left(u_{j}-u\right)\right\rangle \leq 0$, it follows that $F\left(u_{j}\right) \rightarrow F(u)$. 
With $T=I$, we denote briefly $\left(S_{+}\right)_{I}=\left(S_{+}\right),(P M)_{I}=(P M),(Q M)_{I}=(Q M)$ and $(M)_{I}=$ $(M)$. Thus, with $T=I$, we get the standard definitions for classes $\left(S_{+}\right),(P M),(Q M)$ and $(M)$ widely used in the literature.

In this note we will mainly deal with the special case where $T=P$, an orthogonal projection onto a closed subspace $E \subset H$. Define $Q=I-P: H \rightarrow E^{\perp}$. We consider more closely bounded demicontinuous mappings of monotone type with respect to $P$. For instance, according to the previous definitions, $F: H \rightarrow H$ is of class $\left(S_{+}\right)_{P}$, if for any sequence $\left(u_{n}\right)$ such that

$$
u_{n} \rightarrow u, \quad Q u_{n} \longrightarrow Q u, \quad \limsup _{n \rightarrow \infty}\left\langle F\left(u_{n}\right), P\left(u_{n}-u\right)\right\rangle \leq 0
$$

it follows that $u_{j} \rightarrow u$.

The structural properties of the classes $\left(S_{+}\right)_{P},(P M)_{P},(Q M)_{P}$ and $(M)_{P}$, respectively, as well as their mutual relations follow easily from the definitions. The first result is a direct consequence of the definitions.

LeMma 2.1. $(P M)_{E}=(Q M)_{E} \cap(M)_{E}$.

The following comments may clarify the situation for a reader familiar with the general theory of mappings of monotone type:

(i) $F$ is $P$-pseudomonotone if and only if, for any sequence $\left(u_{n}\right)$ such that $(2.1)$ holds, it follows that

$$
\liminf _{n \rightarrow \infty}\left\langle F\left(u_{n}\right), P\left(u_{n}-u\right)\right\rangle \geq\langle F(u), P(u-v)\rangle \quad \forall v \in H
$$

This equivalent definition is in the line of the original definition of pseudomonotone mappings due to $\mathrm{H}$. Brezis; see [5], for instance.

(ii) It is easy to see that $F$ is $P$-quasimonotone, if and only if, for any sequence $\left(u_{n}\right)$ in $H$ such that $u_{n} \rightarrow u$ and $Q u_{n} \rightarrow Q u$, we have

$$
\liminf _{n \rightarrow \infty}\left\langle F\left(u_{n}\right), P\left(u_{n}-u\right)\right\rangle \geq 0
$$

(iii) A direct generalization of the condition $(M)$ used in the study of elliptic problems would be:

$F \in(M)_{P}^{\prime}$ if, for any sequence $\left(u_{n}\right)$ such that $(2.1)$ holds and $F\left(u_{n}\right) \rightarrow w$, it follows that $F(u)=w$.

It is easy to see that, for bounded demicontinuous mappings, $(M)_{P}^{\prime}=(M)_{P}$.

Our main emphasis lies on the classes $\left(S_{+}\right)_{P},(P M)_{P}$ and $(Q M)_{P}$, which have useful properties as will be seen from the following lemmas. Most proofs are omitted.

Lemma 2.2. Let all the mappings considered be bounded and demicontinuous. Then

(1) $\left(S_{+}\right)_{P} \subset(P M)_{P} \subset(Q M)_{P}$.

(2) $\left(S_{+}\right) \subset\left(S_{+}\right)_{P},(P M) \subset(P M)_{P},(Q M) \subset(Q M)_{P}$.

(3) $\left(S_{+}\right)=\left(S_{+}\right)_{P} \Leftrightarrow(P M)=(P M)_{P} \Leftrightarrow(Q M)=(Q M)_{P} \Leftrightarrow \operatorname{dim} E^{\perp}<\infty$. 
Proof. The first two items follow directly from the definitions. If $\operatorname{dim} E^{\perp}<\infty$, then $Q=$ $I-P: H \rightarrow E^{\perp}$ is compact and condition $u_{n} \rightarrow u$ is equivalent to $P u_{n} \rightarrow P u$. Hence it is clear that the class $\left(S_{+}\right)_{P}$ coincide with $\left(S_{+}\right)$with similar conclusion for other classes. On the other hand, the reverse follows from the following simple observation. Assume $\operatorname{dim} E^{\perp}=\infty$ and consider the mapping $F=P-Q$. It is easy to see that $P-Q \in\left(S_{+}\right)_{P} \subset$ $(P M)_{P} \subset(Q M)_{P}$, but $P-Q \notin(Q M)$.

In view of applications, it is useful to notice that any compact mapping is quasimonotone and thus also $P$-quasimonotone. An important property of the classes $\left(S_{+}\right)_{P},(P M)_{P}$ and $(Q M)_{P}$ is that they have conical structure in the following sense.

Lemma 2.3. Let $F: H \rightarrow H$ and $T: H \rightarrow H$ be given bounded demicontinuous mappings. Then we have:

(1) If $F, T \in\left(S_{+}\right)_{P}$, then $F+T \in\left(S_{+}\right)_{P}$ and $\alpha F \in\left(S_{+}\right)_{P}$ for all $\alpha>0$.

(2) If $F, T \in(P M)_{P}$, then $F+T \in(P M)_{P}$ and $\alpha F \in(P M)_{P}$ for all $\alpha \geq 0$.

(3) If $F, T \in(Q M)_{P}$, then $F+T \in(Q M)_{P}$ and $\alpha F \in(Q M)_{P}$ for all $\alpha \geq 0$.

The conical structure of the classes $\left(S_{+}\right)_{P},(P M)_{P}$ and $(Q M)_{P}$ is one of the features that make the classes useful and "nice" to deal with. For the class $(M)_{P}$ the sum of two maps is not necessarily in the same class as we will see.

Another important fact is that the class $\left(S_{+}\right)_{P}$ is stable under quasimonotone perturbations and moreover, the class $(Q M)_{P}$ is, in a sense, a maximal set of such perturbations. Indeed, we have the following lemma.

Lemma 2.4. Let $T: H \rightarrow H$ be a given bounded demicontinuous mapping. Then $T \in(Q M)_{P}$ if and only if $F+T \in\left(S_{+}\right)_{P}$ for all $F \in\left(S_{+}\right)_{P}$.

Proof. If $T \in(Q M)_{P}$ and $F \in\left(S_{+}\right)_{P}$, it is not hard to see that $F+T \in\left(S_{+}\right)_{P}$. On the other hand, assume $F+T \in\left(S_{+}\right)_{P}$ for all $F \in\left(S_{+}\right)_{P}$. Clearly $P \in\left(S_{+}\right)_{P}$ and thus $T+\epsilon P \in\left(S_{+}\right)_{P}$ for all $\epsilon>0$. A contradiction argument shows that necessarily $T \in(Q M)_{P}$.

In view of the degree theory, the conical structure of classes will play a crucial role. Indeed, it is needed to ensure that the family of admissible homotopies will be extensive enough.

The main default of class $(M)_{P}$ is that the sum of two mappings $F_{1}, F_{2} \in(M)_{P}$ does not necessarily remain in the same class. Similarly, if $F_{1} \in(M)_{P}$ and $F_{2} \in\left(S_{+}\right)_{P}$, then it is possible that $F_{1}+F_{2} \notin\left(S_{+}\right)_{P}$. Thus the class $\left(S_{+}\right)_{P}$ does not in general stand perturbation satisfying the condition $(M)_{P}$. These facts can be seen from the next example.

Example 2.5. Let $H$ be a real separable infinite-dimensional Hilbert, $E \subset H$ a closed linear subspace such that $\operatorname{dim} E^{\perp}=\operatorname{dim} E=\infty$. Let $P: H \rightarrow E$ be the orthogonal projection and denote $Q=I-P: H \rightarrow E^{\perp}$. Take $F_{1}=-P$ and

$$
F_{2}(u)=\frac{P u}{\|u\|+1} \quad \forall u \in H
$$

The map $F_{1}=-P$ is weakly continuous and hence $F_{1} \in(M)_{P}$. The map $F_{2}$ is bounded and continuous. We will show that $F_{2} \in\left(S_{+}\right)_{P}$. Assume that $P u_{n} \rightarrow P u, Q u_{n} \rightarrow Q u$ and $\limsup \left\langle F_{2}\left(u_{n}\right), P\left(u_{n}-u\right)\right\rangle \leq 0$. Then limsup $\left\langle P u_{n}, P\left(u_{n}-u\right)\right\rangle \leq 0$ implying $P u_{n} \rightarrow P u$ and 
consequently $F_{2} \in\left(S_{+}\right)_{P}$ and also $F_{2} \in(M)_{P}$. However, the sum $F_{1}+F_{2} \notin(M)_{P}$ and thus $F_{1}+F_{2} \notin\left(S_{+}\right)_{P}$. To see this, let $\left\{e_{n}\right\}$ be an orthonormal basis of $E$ and $u_{n}=e_{1}+e_{n}$. Then, $u_{n} \rightarrow e_{1}:=u$ and

$$
\left(F_{1}+F_{2}\right)\left(u_{n}\right)=-\left(e_{1}+e_{n}\right)+\frac{e_{1}+e_{n}}{\left\|e_{1}+e_{n}\right\|+1}=-\frac{\sqrt{2}}{1+\sqrt{2}}\left(e_{1}+e_{n}\right) .
$$

Thus $\left(F_{1}+F_{2}\right)\left(u_{n}\right) \rightarrow-(\sqrt{2} /(1+\sqrt{2})) e_{1}:=w$ and

$$
\limsup \left\langle\left(F_{1}+F_{2}\right)\left(u_{n}\right), u_{n}-u\right\rangle=\lim \left(-\frac{\sqrt{2}}{1+\sqrt{2}}\left\langle e_{1}+e_{n}, e_{n}\right\rangle\right)=-\frac{\sqrt{2}}{1+\sqrt{2}}<0 .
$$

But, $\left(F_{1}+F_{2}\right)(u)=\left(F_{1}+F_{2}\right)\left(e_{1}\right)=-e_{1} / 2 \neq w=-(\sqrt{2} /(1+\sqrt{2})) e_{1}$ and, consequently, $F_{1}+F_{2} \notin(M)_{P}$.

\section{Construction of the degree}

Let $H$ be a real separable Hilbert space, $E$ a closed subspace of $H$ and $P: H \rightarrow E$ the corresponding orthogonal projection. As before, we denote $Q=I-P: H \rightarrow E^{\perp}$. We consider a family of mappings

$$
\mathscr{F}_{G}=\{F=Q(I-C)+P N: \bar{G} \longrightarrow H\},
$$

where $G$ is an open bounded set in $H, C$ is compact and $N$ is a bounded demicontinuous mapping of class $\left(S_{+}\right)_{P}$. Let

$$
\mathscr{F}=\left\{F \in \mathscr{F}_{G} \mid \mathrm{G} \text { is open and bounded }\right\} .
$$

Since any Leray-Schauder type map is of class $\left(S_{+}\right) \subset\left(S_{+}\right)_{P}$, we have $I-C=Q(I-C)+$ $P(I-C) \in \mathscr{F}$, that is, $\mathscr{F}$ contains the Leray-Schauder type maps. We will construct the degree theory for the class $\mathscr{F}$, which will be a unique extension of the classical LeraySchauder degree in Hilbert space. Note that in case $E$ is finite dimensional, the projection $P$ is compact, and we can write

$$
F=Q(I-C)+P N=I-(P+Q C-P N),
$$

which is of the Leray-Schauder type. Hence, $\mathscr{F}$ reduces to the class of Leray-Schauder type maps whenever $\operatorname{dim} E<\infty$. If $\operatorname{dim} E^{\perp}<\infty$, then $Q$ is compact and any $F \in \mathscr{F}_{F}$ can be written in the form

$$
F=Q(I-C)+P N=N+Q(I-C-N)
$$

which is of class $\left(S_{+}\right)$by Lemmas 2.2 and 2.4 (recall that compact maps are quasimonotone). Hence $\mathscr{F}_{F}$ consists of $\left(S_{+}\right)$-type maps, only, whenever $\operatorname{dim} E^{\perp}<\infty$. Conversely, if $F$ : $\bar{G} \rightarrow H$ is a bounded, demicontinuous map of class $\left(S_{+}\right)_{P}$ and $\operatorname{dim} E^{\perp}<\infty$, then $\left(S_{+}\right)_{P}=$ $\left(S_{+}\right), Q$ is compact and

$$
F=Q(I-Q(I-F))+P F \in \mathscr{F} .
$$

The degree for mappings of class $\left(S_{+}\right)$can be found in [7] in a more general setting. 
Hereafter, we are mainly interested in the case where both $E$ and $E^{\perp}$ are infinite dimensional. The construction of the new degree can be done by using the Galerkin approximation with respect to the space $E$ (cf. [3]). Note that the Q-component, which is of the Leray-Schauder type, needs no approximation. However, we will give here another, shorter, construction which is based on linear compact mappings. The method is a simplified version of the so called "elliptic super-regularization" used in a more general context elsewhere (see [4]).

Following that approach, let $\psi: E \rightarrow E$ be some fixed compact linear selfadjoint injection. To any $F=Q(I-C)+P N \in \mathscr{F}_{G}$, we associate a family of mappings defined by

$$
F_{\lambda}=I-Q C+\lambda \psi^{2} P N, \quad \text { where } \lambda>0 .
$$

Clearly $F_{\lambda}: \bar{G} \rightarrow H$ is a Leray-Schauder type map. We denote by $\mathrm{d}_{L S}\left(F_{\lambda}, G, 0\right)$ the corresponding Leray-Schauder degree (which is well-defined only if $0 \notin F_{\lambda}(\partial G)$ ). We start with the following basic result.

Lemma 3.1. Assume that $F=Q(I-C)+P N \in \mathscr{F}_{G}, G \subset H$ being open and bounded, and that $0 \notin F(\partial G)$. Then, there exists $\lambda_{0} \geq 0$ such that $0 \notin F_{\lambda}(\partial G)$, and $d_{L S}\left(F_{\lambda}, G, 0\right)$ is constant in $\lambda$, for all $\lambda>\lambda_{0}$.

Proof. We will argue by contradiction. Assume that the first assertion is not valid. Then we can find sequences $\left(\lambda_{n}\right), \lambda_{n} \rightarrow \infty,\left(u_{n}\right) \subset \partial G$ such that $F_{\lambda_{n}}\left(u_{n}\right)=0$. At least for subsequences, we can write $u_{n} \rightarrow u, N\left(u_{n}\right) \rightarrow w$ and $C\left(u_{n}\right) \rightarrow z$. The equation $F_{\lambda_{n}}\left(u_{n}\right)=0$ is equivalent to

$$
\begin{aligned}
Q u_{n}-Q C\left(u_{n}\right) & =0 \\
P u_{n}+\lambda_{n} \psi^{2} P N\left(u_{n}\right) & =0
\end{aligned}
$$

and thus $Q u_{n} \rightarrow Q u=Q z$ and $\psi^{2} P N\left(u_{n}\right) \rightarrow 0=\psi^{2} P w$ implying $P w=0$. Hence,

$$
\begin{aligned}
\limsup \left\langle N\left(u_{n}\right), P\left(u_{n}-u\right)\right\rangle & =\limsup \left\langle P N\left(u_{n}\right), P u_{n}-P u\right\rangle \\
& =\limsup \left\langle P N\left(u_{n}\right),-\lambda_{n} \psi^{2} P N\left(u_{n}\right)\right\rangle \\
& =\limsup \left\{-\lambda_{n}\left\|\psi P N\left(u_{n}\right)\right\|^{2}\right\} \leq 0,
\end{aligned}
$$

which implies $u_{n} \rightarrow u \in \partial G$, since $N \in\left(S_{+}\right)_{P}$. Consequently, we have $F(u)=Q(u-C(u))+$ $P N(u)=0$ giving a contradiction. The second assertion follows from the homotopy invariance property of $\mathrm{d}_{L S}$. Indeed, let $\lambda_{2}>\lambda_{1}>\lambda_{0}$. Then $F_{\lambda}, \lambda_{1} \leq \lambda \leq \lambda_{2}$, defines a LeraySchauder type homotopy such that $F_{\lambda}(u) \neq 0$ for all $\lambda_{1} \leq \lambda \leq \lambda_{2}$ and $u \in \partial G$. Hence

$$
\mathrm{d}_{L S}\left(F_{\lambda_{1}}, G, 0\right)=\mathrm{d}_{L S}\left(F_{\lambda_{2}}, G, 0\right)
$$

and since $\lambda_{1}>\lambda_{0}$ and $\lambda_{2}>\lambda_{0}$ were arbitrary, the second assertion is proved.

It is relevant to define a new integer-valued function $\mathrm{d}$ by setting

$$
\begin{aligned}
& \mathrm{d}(F, G, 0)=\lim _{\lambda \rightarrow \infty} \mathrm{d}_{L S}\left(F_{\lambda}, G, 0\right), \quad \text { if } 0 \notin F(\partial G), \\
& \mathrm{d}(F, G, h)=\mathrm{d}(F-h, G, 0) \quad \text { for any } h \notin F(\partial G) .
\end{aligned}
$$


We will show in our next section that the integer-valued function d satisfies the conditions of the classical topological degree.

\section{Properties and uniqueness of the degree}

Throughout the next steps we assume that $F \in \mathscr{F}_{G}$, where $G \subset H$ is an open bounded set and $h \notin F(\partial G)$. We will verify four conditions (a) to (d) - existence of solutions, additivity of domains, normalization, and invariance under homotopies. In fact, it is easy to see that (a) is a consequence of (b). The main argument used to establish the properties of the degree will be the following: for any closed set $A \subset \bar{G}$ with $h \notin F(A)$ there exists $\lambda_{0}>0$ such that

$$
0 \notin(F-h)_{\lambda}(A) \quad \forall \lambda>\lambda_{0}
$$

The proof of (4.1) is an obvious variant of the first part of the proof of Lemma 3.1; it suffices to replace the boundary $\partial G$ by the closed set $A$.

(a) If $\mathrm{d}(F, G, h) \neq 0$, then there exists a solution for the equation $F(u)=h$ in $G$.

(b) (Additivity of domains) Let $G_{1}$ and $G_{2}$ be a pair of disjoint open subsets of $G$ such that $h \notin F\left(\bar{G} \backslash\left(G_{1} \cup G_{2}\right)\right)$. Then,

$$
\mathrm{d}(F, G, h)=\mathrm{d}\left(F, G_{1}, h\right)+\mathrm{d}\left(F, G_{2}, h\right) .
$$

(c) (Normalizing map) The normalizing map is the identity $I=Q+P$. If $h \in G$, where $G \subset H$ is an open bounded set, we have $\mathrm{d}(I, G, h)=+1$.

The above properties of the degree $d$ are easily deduced from the corresponding properties of the Leray-Schauder degree, using (4.1). We give more details for the property of invariance under a homotopy.

(d) (Invariance under homotopy) We say that a mapping $N:[0,1] \times \bar{G} \rightarrow H$ is $a$ bounded homotopy of class $\left(S_{+}\right)_{P}$, if it is bounded, demicontinuous, and the conditions

$$
u_{n} \rightarrow u, Q u_{n} \rightarrow Q u, t_{n} \longrightarrow t, \quad \limsup _{n \rightarrow \infty}\left\langle N\left(t_{n}, u_{n}\right), P\left(u_{n}-u\right)\right\rangle \leq 0
$$

imply $u_{n} \rightarrow u$ and $N\left(t_{n}, u_{n}\right) \rightarrow N(t, u)$. Let $C:[0,1] \times \bar{G} \rightarrow H$ be compact and let

$$
F(t, u)=Q(u-C(t, u))+P N(t, u), \quad 0 \leq t \leq 1, u \in \bar{G}
$$

where $N$ is a bounded homotopy of class $\left(S_{+}\right)_{P}$. The family of admissible homotopies is

$$
\mathscr{H}_{G}=\{F:[0,1] \times \bar{G} \longrightarrow H \mid F \text { is given by }(4.4)\} .
$$

Denote

$$
\mathscr{H}=\left\{F \in \mathscr{H}_{G} \mid \mathrm{G} \text { is open and bounded }\right\} .
$$


Let $\left\{h_{t}: 0 \leq t \leq 1\right\}$ be a continuous curve in $H$ such that $h_{t} \notin F(t, \partial G)$ for all $t \in[0,1]$, where $F \in \mathscr{H}_{G}$. Then

$$
\mathrm{d}\left(F(t, \cdot), G, h_{t}\right) \text { is constant in } t \text { on }[0,1] \text {. }
$$

We will prove (4.7). Without loss of generality we can assume that $h_{t} \equiv 0$ by absorbing $h_{t}$ into $F(t, x)$. We omit the proof of the following lemma, which is a straightforward generalization of the proof of Lemma 3.1.

Lemma 4.1. Assume that $F=Q(I-C)+P N \in \mathscr{H}_{G}, G \subset H$ is open and bounded and $0 \notin$ $F(t, \partial G)$ for all $0 \leq t \leq 1$. Then there exists $\lambda_{0}>0$ such that $0 \notin F_{\lambda}(t, \partial G)$ for all $0 \leq t \leq 1$ and $\lambda>\lambda_{0}$.

Clearly, for a fixed $\lambda>\lambda_{0}, F_{\lambda}(t, \cdot), 0 \leq t \leq 1$, defines a Leray-Schauder type homotopy (recall that $\left.F_{\lambda}(t, u)=u-Q C(t, u)+\lambda \psi^{2} P N(t, u)\right)$. Hence, according to Lemma 3.1,

$$
\mathrm{d}(F(t, \cdot), G, 0)=\mathrm{d}_{L S}\left(F_{\lambda}(t, \cdot), G, 0\right) \text { is constant } \quad \forall 0 \leq t \leq 1, \lambda>\lambda_{0},
$$

which proves (4.7). In view of applications it is essential to notice the following Lemma 4.2. Let $F, T \in \mathscr{F}_{G}$, where $G$ is an open bounded set. Then the affine homotopy

$$
F(t, \cdot)=(1-t) F+t T, \quad 0 \leq t \leq 1
$$

is admissible, that is, it belongs to $\mathscr{H}_{G}$.

The uniqueness of the degree $d$ will be a consequence of the uniqueness of the LeraySchauder degree.

THEOREM 4.3. There exists one and only one degree function for the class $\mathscr{F}$ satisfying properties (a), (b) and (d), which is invariant under the homotopy class $\mathcal{H}$.

Proof. The existence of the degree is already established. Let $\mathrm{d}$ be given by (3.10). Assume that $\mathrm{d}_{1}$ is another degree function satisfying conditions (a)-(d) above. Let $G \subset X$ be any open bounded set and $F=Q(I-C)+P N$ any mapping in $\mathscr{F}_{G}$. It is sufficient to prove that

$$
\mathrm{d}_{1}(F, G, 0)=\mathrm{d}(F, G, 0),
$$

assuming $0 \notin F(\partial G)$. By definition there exists $\lambda_{0}>0$ such that

$$
\mathrm{d}(F, G, 0)=\mathrm{d}_{L S}\left(F_{\lambda}, G, 0\right) \quad \forall \lambda>\lambda_{0},
$$

where $F_{\lambda}=I-Q C+\lambda \psi^{2} P N$ and $\psi: E \rightarrow E$ is a (arbitrary but fixed) linear self-adjoint compact injection.

We know that the Leray-Schauder degree is unique and any Leray-Schauder type map $I-C$ is of class $\left(S_{+}\right)_{P}$. It is easy to see that defining

$$
\hat{\mathrm{d}}_{L S}(I-C, D, y)=\mathrm{d}_{1}(I-C, D, y)
$$


for any $y \in H \backslash(I-C)(\partial D)$, where $D \subset H$ is an open bounded set, gives a degree function for the Leray-Schauder type mappings in $H$. By the uniqueness of the Leray-Schauder degree $\hat{\mathrm{d}}_{L S}=\mathrm{d}_{L S}$ and thus

$$
\mathrm{d}_{L S}(I-C, D, y)=\mathrm{d}_{1}(I-C, D, y) .
$$

In particular,

$$
\mathrm{d}_{L S}\left(F_{\lambda}, G, 0\right)=\mathrm{d}_{1}\left(F_{\lambda}, G, 0\right) \quad \forall \lambda>\lambda_{0} .
$$

The proof will be completed by showing that there exists $\lambda_{1} \geq \lambda_{0}$ such that

$$
(1-t) F(u)+t F_{\lambda}(u) \neq 0 \quad \forall u \in \partial G, 0 \leq t \leq 1, \lambda \geq \lambda_{1}
$$

implying

$$
\mathrm{d}_{1}\left(F_{\lambda}, G, 0\right)=\mathrm{d}_{1}(F, G, 0) \quad \forall \lambda \geq \lambda_{1} .
$$

This is done by the same arguments as in Lemma 3.1

\section{On the continuation method}

The standard application of the topological degree theory in order to obtain existence results is through the use of the homotopy invariance property. Hence a typical, and in fact the most useful, form of the continuation method based on affine homotopy can be stated as follows.

Theorem 5.1. Let $F, T \in \mathscr{F}_{G}$, where $G$ is an open bounded set. Assume that $(1-t) h \notin$ $[(1-t) F+t T](\partial G)$ for all $0 \leq t \leq 1$. Then,

$$
d(F, G, h)=d(T, G, 0) .
$$

Moreover, if $d(T, G, 0) \neq 0$, the equation $F(u)=h$ admits at least one solution $u \in G$.

The underlying idea is to replace the equation $F(u)=h$ which is "hard to solve" by some equation $T(u)=0$ which is "easy to solve". Note that in practice $F$ and $T$ are somehow connected, the easy problem $T(u)=0$ being a simplification (e.g. linearization) of the hard one.

To illuminate the general argumentation, we consider the semilinear equation

$$
L u-N(u)=0, \quad u \in D(L),
$$

where $L: D(L) \subset H \rightarrow H$ is a linear, densely defined, closed operator with $\operatorname{Im} L=(\operatorname{Ker} L)^{\perp}$ and $N: H \rightarrow H$ a nonlinearity. The inverse $K: \operatorname{Im} L \rightarrow \operatorname{Im} L$ of the restriction of $L$ to $\operatorname{Im} L \cap$ $D(L)$ is assumed to be compact. It is easy to see that (5.2) can be written equivalently as

$$
Q(u-K Q N(u))+P N(u)=0, \quad u \in H,
$$

where $P: H \rightarrow \operatorname{Ker} L$ and $Q=I-P: H \rightarrow \operatorname{Im} L$ are orthogonal projections. Above we have used the fact that $K Q-P$ is the right inverse of $L-P$. In case $N \in\left(S_{+}\right)_{P}$ the mapping 
$F=Q(I-K Q N)+P N \in \mathscr{F}$ and the degree theoretic approach is possible. As an example, we present the following generalization of a continuation theorem of Mawhin $[9,10]$.

Theorem 5.2. Let $L, N$ and $G$ be as indicated above. Assume that

(i) $0 \notin(L-t N)(\partial G \cap D(L))$ for all $t \in(0,1]$,

(ii) $P N u \neq 0$, for all $u \in \operatorname{Ker} L \cap \partial G$.

Then,

$$
d(F, G, 0)=d\left(P N_{\mid \operatorname{Ker} L}, G \cap \operatorname{Ker} L, 0\right) .
$$

Notice that, since $N$ is of class $\left(S_{+}\right)_{P}, P N_{\mid \operatorname{Ker} L}: \operatorname{Ker} L \rightarrow \operatorname{Ker} L$ is of class $\left(S_{+}\right)$; hence, $\mathrm{d}\left(P N_{\mid \operatorname{Ker} L}, G \cap \operatorname{Ker} L, 0\right)$ is well-defined, although $\operatorname{Ker} L$ can be infinite dimensional.

Proof. Now $E=\operatorname{Ker} L$. Let $\psi: E \rightarrow E$ be a fixed linear self-adjoint compact injection. By definition,

$$
\mathrm{d}(F, G, 0)=\lim _{\lambda \rightarrow \infty} \mathrm{d}_{L S}\left(I-K Q N+\lambda \psi^{2} P N, G, 0\right) .
$$

Arguing as in Lemma 3.1, we can show that there exists $\lambda_{0}>0$ such that, for $\lambda \geq \lambda_{0}, t \in$ $[0,1], u \in \partial G$

$$
u-t K Q N u+\lambda \psi^{2} P N(u) \neq 0 .
$$

Consequently, by Theorem $5.1, \lim _{\lambda \rightarrow \infty} \mathrm{d}_{L S}\left(I-t K Q N+\lambda \psi^{2} P N, G, 0\right)$ is independent of $t \in[0,1]$. Therefore, by Leray's product theorem,

$$
\begin{aligned}
\mathrm{d}(F, G, 0) & =\lim _{\lambda \rightarrow \infty} \mathrm{d}_{L S}\left(I+\lambda \psi^{2} P N, G, 0\right) \\
& =\lim _{\lambda \rightarrow \infty} \mathrm{d}_{L S}\left(I_{\mid \operatorname{Ker} L}+\lambda \psi^{2} P N_{\mid \operatorname{Ker} L}, G \cap \operatorname{Ker} L, 0\right) \\
& =\mathrm{d}\left(P N_{\mid \operatorname{Ker} L}, G \cap \operatorname{Ker} L, 0\right) .
\end{aligned}
$$

The next lemma is an immediate generalization of a classical result about linear operators. Let $L$ be as above. Assume that $B: H \rightarrow H$ is linear bounded operator such that $B \in\left(S_{+}\right)_{P}$ and $L-B$ is injective. Then $T=Q(I-K Q B)+P B \in \mathscr{F}$ is linear and injective.

Lemma 5.3. Let $B: H \rightarrow H$ be a linear bounded operator such that $B \in\left(S_{+}\right)_{P}$ and $L-B$ is injective. Then for any open bounded set $G$,

$$
d(T, G, y) \neq 0 \quad \text { for any } y \in T(G) .
$$

Proof. For $y \in T(G)$ we have $y=T v$ for some unique $v \in H$. To prove (5.8) we notice first, by the injectivity of $T$ and the additivity of degree, that

$$
\mathrm{d}(T, G, y)=\mathrm{d}(T, B(0, R), y) \quad \text { for any } R>\|v\| .
$$

It is clear that $T u \neq t y$ for all $\|u\|=R$ and $0 \leq t \leq 1$. Hence $\mathrm{d}(T, B(0, R), t y)$ is constant in $t \in[0,1]$ and consequently

$$
\mathrm{d}(T, B(0, R), y)=\mathrm{d}(T, B(0, R), 0) .
$$


Since $B$ is linear, the mapping $T$ is linear and thus odd. The same is true for the approximation $T_{\lambda}=I-Q K Q B+\lambda \psi^{2} P B$ used in the construction of the degree. By the standard property of the Leray-Schauder degree we then obtain

$$
\mathrm{d}(T, B(0, R), 0)=\lim _{\lambda \rightarrow \infty} \mathrm{d}_{L S}\left(T_{\lambda}, B(0, R), 0\right), \text { an odd integer, }
$$

completing the proof.

The next lemma may be helpful for the verification of one of the conditions about the linear map $B$ in Lemma 5.3.

Lemma 5.4. Let $B: H \rightarrow H$ be a linear bounded operator. Then $B \in\left(S_{+}\right)_{P}$ if and only if $P B_{\mid \operatorname{Ker} L}: \operatorname{Ker} L \rightarrow \operatorname{Ker} L$ is of class $\left(S_{+}\right)$.

Proof. Assume that $P B_{\mid \operatorname{Ker} L}: \operatorname{Ker} L \rightarrow \operatorname{Ker} L$ is of class $\left(S_{+}\right)$. Let $u_{n} \rightarrow u, Q u_{n} \rightarrow Q u$ and

$$
\limsup \left\langle B u_{n}, P\left(u_{n}-u\right)\right\rangle \leq 0
$$

Since $B Q u_{n} \rightarrow B Q u$ we have

$$
\limsup \left\langle P B P u_{n}, P u_{n}-P u\right\rangle \leq 0
$$

implying $P u_{n} \rightarrow P u$, which completes the first part of the proof. On the other hand, if $B \in\left(S_{+}\right)_{P}$, then clearly $P B_{\mid \operatorname{Ker} L} \in\left(S_{+}\right)$.

\section{Semilinear systems of equations}

In this section we will provide abstract examples, which will show that the degree theory constructed in this paper has practical value in view of applications. The aim is to consider the nature of mappings which are of class $\left(S_{+}\right)_{P}$, but not necessarily of class $\left(S_{+}\right)$. For the general treatment of semilinear problems we refer to $[6,8]$.

Let $V$ be a real separable Hilbert space and denote $H=V^{n}$ with $n \geq 2$. For $k=1,2, \ldots$, $n$, let $L_{k}: D\left(L_{k}\right) \subset V \rightarrow V$ be a linear, densely defined, closed operator with $\operatorname{Im} L_{k}=$ $\left(\operatorname{Ker} L_{k}\right)^{\perp}$. The inverse $K_{k}: \operatorname{Im} L_{k} \rightarrow \operatorname{Im} L_{k}$ of the restriction of each $L_{k}$ to $\operatorname{Im} L_{k} \cap D\left(L_{k}\right)$ is assumed to be a compact linear operator. We define the diagonal operator $L: D(L) \subset$ $V^{n} \rightarrow V^{n}$ by setting

$$
L u=\left(L_{1} u_{1}, L_{2} u_{2}, \ldots, L_{n} u_{n}\right), \quad u=\left(u_{1}, u_{2}, \ldots, u_{n}\right) \in D(L),
$$

where $D(L)=D\left(L_{1}\right) \times D\left(L_{2}\right) \times \cdots \times D\left(L_{n}\right)$. The inverse $K=L^{-1}: \operatorname{Im} L \rightarrow \operatorname{Im} L$ is compact, with $K u=\left(K_{1} u_{1}, \ldots, K_{n} u_{n}\right)$ for $u=\left(u_{1}, u_{2}, \ldots, u_{n}\right) \in \operatorname{Im} L$. We denote by $P$ and $Q$ the orthogonal projections onto $\operatorname{Ker} L$ and $\operatorname{Im} L$, respectively. Thus, with obvious notations, we can write

$$
P u=\left(P_{1} u_{1}, P_{2} u_{2}, \ldots, P_{n} u_{n}\right), \quad Q u=\left(Q_{1} u_{1}, Q_{2} u_{2}, \ldots, Q_{n} u_{n}\right)
$$


for any $u=\left(u_{1}, u_{2}, \ldots, u_{n}\right) \in V^{n}$. Let $N: V^{n} \rightarrow V^{n}$ be a (possibly nonlinear) bounded demicontinuous map. Then the equation

$$
L u-N(u)=h, \quad u \in D(L),
$$

can be written equivalently as

$$
Q(u-K Q N(u))+P N(u)=\hat{h}, \quad u \in V^{n},
$$

where $\hat{h}=(K Q-P) h$. If $N$ is bounded, demicontinuous and of class $\left(S_{+}\right)_{P}$, we can apply the degree theory constructed in Section 3.

Assume that $\operatorname{dim} \operatorname{Ker} L_{k}=\infty$ for $k=1,2, \ldots, p$ for some $p \in\{1, \ldots, n\}$, and that $\operatorname{dim}$ $\operatorname{Ker} L_{k}<\infty$ for $k=p+1, \ldots, n$. For a mapping $N: V^{n} \rightarrow V^{n}$, where

$$
N(u)=\left(N_{1}(u), N_{2}(u), \ldots, N_{n}(u)\right),
$$

we use the notation $u=(v, z) \in V^{p} \times V^{n-p}$ and

$$
\mathcal{N}_{p}(v, z)=\left(N_{1}(v, z), N_{2}(v, z), \ldots, N_{p}(v, z)\right) .
$$

The following lemma provides conditions under which $N$ is of class $\left(S_{+}\right)_{P}$ giving the possibility to use the degree defined in Section 3 to solve (6.3).

Lemma 6.1. let $N: V^{n} \rightarrow V^{n}$ be bounded and demicontinuous. Assume that

(i) for each $z \in V^{n-p}$ the mapping $\mathcal{N}_{p}(\cdot, z): V^{p} \rightarrow V^{p}$ is of class $\left(S_{+}\right)$.

(ii) $\mathcal{N}_{p}(v, \cdot): V^{n-p} \rightarrow V^{p}$ is continuous, uniformly for $v$ in any bounded set $B \subset V^{p}$.

Then $N \in\left(S_{+}\right)_{P}$.

Proof. Let $\left(u^{(j)}\right) \subset V^{n}$ be a sequence such that

$$
u^{(j)} \rightarrow u, \quad Q u^{(j)} \longrightarrow Q u, \quad \limsup _{j \rightarrow \infty}\left\langle N\left(u^{(j)}\right), P\left(u^{(j)}-u\right)\right\rangle \leq 0 .
$$

Denote $u^{(j)}=\left(v^{(j)}, z^{(j)}\right)$, where $v^{(j)}=\left(u_{1}^{(j)}, u_{2}^{(j)}, \ldots, u_{p}^{(j)}\right)$ and $z^{(j)}=\left(u_{p+1}^{(j)}, u_{p+2}^{(j)}, \ldots, u_{n}^{(j)}\right)$. Similarly, let $u=(v, z)=\left(u_{1}, u_{2}, \ldots, u_{n}\right)$. By our assumptions on $L$, we have $z^{(j)} \rightarrow z$ in $V^{n-P}$. Since $N$ is bounded, we thus have

$$
\lim _{j \rightarrow \infty}\left\langle N_{k}\left(v^{(j)}, z^{(j)}\right), P_{k}\left(u_{k}^{(j)}-u_{k}\right)\right\rangle=0 \quad \forall k=p+1, p+2, \ldots, n .
$$

Consequently,

$$
\limsup _{j \rightarrow \infty}\left\langle N\left(v^{(j)}, z^{(j)}\right), P\left(u^{(j)}-u\right)\right\rangle=\limsup _{j \rightarrow \infty}\left\langle\mathcal{N}_{p}\left(v^{(j)}, z^{(j)}\right), v^{(j)}-v\right\rangle .
$$

Since by (ii)

$$
\limsup _{j \rightarrow \infty}\left\langle\mathcal{N}_{p}\left(v^{(j)}, z^{(j)}\right), v^{(j)}-v\right\rangle=\limsup _{j \rightarrow \infty}\left\langle\mathcal{N}_{p}\left(v^{(j)}, z\right), v^{(j)}-v\right\rangle,
$$


we finally get

$$
\limsup _{j \rightarrow \infty}\left\langle\mathcal{N}_{p}\left(v^{(j)}, z\right), v^{(j)}-v\right\rangle=\limsup _{j \rightarrow \infty}\left\langle\mathcal{N}\left(v^{(j)}, z^{(j)}\right), P\left(u^{(j)}-u\right)\right\rangle \leq 0 .
$$

By assumption (i), we obtain $v^{(j)} \rightarrow v$ in $V^{p}$ and hence $u^{(j)} \rightarrow u$.

The main point in the previous lemma is that there is no monotonicity-type hypothesis on the components $N_{p+1}, \ldots, N_{n}$. Recall also that $\mathcal{N}_{p}(\cdot, z): V^{p} \rightarrow V^{p}$ is of class $\left(S_{+}\right)$if it is strongly monotone. Therefore, Lemma 6.1 can be applied for instance to the system

$$
\begin{gathered}
L_{1} v-N_{1,1}(v)-N_{1,2}(z)=h_{1}, \\
L_{2} z-N_{2}(v, z)=h_{2},
\end{gathered}
$$

with $\operatorname{dim} \operatorname{Ker} L_{1}=\infty$ and $\operatorname{dim} \operatorname{Ker} L_{2}<\infty$. Assuming that $N_{1,1}, N_{1,2}$ and $N_{2}$ are bounded and demicontinuous, that $N_{1,1}$ is strongly monotone and $N_{1,2}$ continuous, it is easy to show that $N: V^{2} \rightarrow V^{2}: u=(v, z) \mapsto\left(N_{1,1}(v)+N_{1,2}(z), N_{2}(v, z)\right)$ satisfies the conditions of Lemma 6.1. Hence, the mapping $N: V^{2} \rightarrow V^{2}$ is of class $\left(S_{+}\right)_{P}$. It is not hard to see however that $N$ is not necessarily of class $\left(S_{+}\right)$, due to the liberality of the hypotheses imposed on $\mathrm{N}_{2}$.

In the next lemma, we use slightly different hypotheses.

Lemma 6.2. Let $N: V^{n} \rightarrow V^{n}$ be bounded and demicontinuous. Let $\mathcal{N}_{p}: V^{n} \rightarrow V^{p}$ be defined as before. Assume that $\mathcal{N}_{p}(v, \cdot): V^{n-p} \rightarrow V^{p}$ is continuous for all $v \in V^{p}$, and that, for each $z^{\prime} \in V^{n-p}$, there exist $\delta=\delta\left(z^{\prime}\right)>0$ and a constant $c=c\left(z^{\prime}\right)>0$ such that

$$
\left\langle\mathcal{N}_{p}(v, z)-\mathcal{N}_{p}\left(v^{\prime}, z\right), v-v^{\prime}\right\rangle \geq c\left\|v-v^{\prime}\right\|^{2} \quad \forall v, v^{\prime} \in V^{p}, z \in B\left(z^{\prime}, \delta\right) .
$$

Then, $N \in\left(S_{+}\right)_{P}$.

Proof. Let $\left(u^{(j)}\right) \subset V^{n}$ be a sequence such that

$$
u^{(j)} \rightarrow u, \quad Q u^{(j)} \longrightarrow Q u, \quad \limsup _{j \rightarrow \infty}\left\langle N\left(u^{(j)}\right), P\left(u^{(j)}-u\right)\right\rangle \leq 0
$$

As in the proof of Lemma 6.1 (and with same notations), we get $z^{(j)} \rightarrow z$ in $V^{n-p}$ and

$$
\limsup _{j \rightarrow \infty}\left\langle\mathcal{N}_{p}\left(v^{(j)}, z^{(j)}\right), v^{(j)}-v\right\rangle=\limsup _{j \rightarrow \infty}\left\langle N\left(v^{(j)}, z^{(j)}\right), P\left(u^{(j)}-u\right)\right\rangle \leq 0 .
$$

Hence,

$$
\underset{j \rightarrow \infty}{\limsup }\left\langle\mathcal{N}_{p}\left(v^{(j)}, z^{(j)}\right)-\mathcal{N}_{p}\left(v, z^{(j)}\right), v^{(j)}-v\right\rangle \leq 0
$$

By condition $(6.13), v^{(j)} \rightarrow v$ in $V^{p}$ and consequently $u^{(j)} \rightarrow u$. 
The previous lemma suggests different types of application.

Example 6.3. With $V=L^{2}(\Omega), \Omega \subset \mathbb{R}^{N}$, let $n=2, p=1$. Consider a system (6.3) in $V^{2}$. Let $N: V^{2} \rightarrow V^{2}$ be a Nemytski operator generated by a function $g: \Omega \times \mathbb{R}^{2} \rightarrow \mathbb{R}^{2}$, satisfying the usual Carathéodory conditions, together with a linear growth condition. Assume that $g(x, s, t)=\left(g_{1}(x, s, t), g_{2}(x, s, t)\right)$, where $g_{1}$ is of the form

$$
g_{1}(x, s, t)=q(x, t) r(x, s) \quad \forall(x, s, t) \in \Omega \times \mathbb{R}^{2},
$$

and that there exist constants $b>a>0$ and $c>0$ such that

(i) $0<a \leq q(x, t) \leq b$ for a. a. $x \in \Omega$ and all $t \in \mathbb{R}$,

(ii) $\left(r(x, s)-r\left(x, s^{\prime}\right)\right)\left(s-s^{\prime}\right) \geq c\left|s-s^{\prime}\right|^{2}$ for a. a. $x \in \Omega$ and all $s, s^{\prime} \in \mathbb{R}$.

No further restrictions are imposed on $g_{2}$. Then, it is easy to see that the conditions of Lemma 6.2 are satisfied and $N \in\left(S_{+}\right)_{P}$.

Example 6.4. As before, assume that $\operatorname{dim} \operatorname{Ker} L_{k}=\infty$ for $k=1,2, \ldots, p$ for some $p \in$ $\{1, \ldots, n\}$, and that $\operatorname{dim} \operatorname{Ker} L_{k}<\infty$ for $k=p+1, \ldots, n$. Consider (6.3) with $N$ being now a linear map, denoted by $\mathcal{A}$, of the following type. Let $A=\left(a_{l k}\right)$ be a real $n \times n$-matrix and $\mathscr{A}: V^{n} \rightarrow V^{n}$ the constant multiplication operator induced by $A$, that is, for any $u=\left(u_{1}, u_{2}, \ldots, u_{n}\right) \in V^{n}$

$$
\mathscr{A} u=w=\left(w_{1}, w_{2}, \ldots, w_{n}\right)
$$

with $w_{l}=\sum_{k=1}^{n} a_{l k} u_{k}, l=1,2, \ldots, n$. Under the mild positivity condition, that the matrix $\left(a_{l k}\right)_{l, k=1}^{p}$ is positive definite, it is easy to show, using Lemma 6.2, that $\mathscr{A} \in\left(S_{+}\right)_{P}$ (cf. [1] for a different treatment). Note that for $p=1$ we assume only $a_{11}>0$.

\section{Semilinear problems with a non-symmetric linear part}

So far we have considered only the class $\left(S_{+}\right)_{T}$, where $T=P$. We close this note by an observation, which shows that also different choices of $T$ may be relevant. Let $T=\mathscr{E} P$, where $\mathscr{E}: H \rightarrow H$ is a linear homeomorphism and $P$ an orthogonal projection. Then $\operatorname{Ker} T=\operatorname{Ker} P$ and $N \in\left(S_{+}\right)_{\mathscr{~} P}$ if and only if $\mathscr{F}^{*} N \in\left(S_{+}\right)_{P}$. A similar observation holds for $\mathscr{L} P$-pseudomonotone and $\mathscr{E} P$-quasimonotone mappings. Such an operator $T$ has been used in [2] to obtain existence results for semilinear equations with a non-symmetric linear part.

Let $L: D(L) \subset H \rightarrow H$ be a densely defined closed linear operator with closed range $\operatorname{Im} L$. Then the adjoint $L^{*}: D\left(L^{*}\right) \subset H \rightarrow H$ of $L$ inherits these properties, that is, also $L^{*}$ is a densely defined closed linear operator having closed range. Since

$$
\operatorname{Im} L^{*}=(\operatorname{Ker} L)^{\perp}, \quad \operatorname{Im} L=\left(\operatorname{Ker} L^{*}\right)^{\perp},
$$

the space $H$ has the orthogonal direct sum decompositions

$$
H=\operatorname{Ker} L \oplus \operatorname{Im} L^{*}=\operatorname{Ker} L^{*} \oplus \operatorname{Im} L
$$

Denote the corresponding orthogonal projections by $P: H \rightarrow \operatorname{Ker} L, \tilde{P}=I-P: H \rightarrow \operatorname{Im} L^{*}$, 
$Q: H \rightarrow \operatorname{Ker} L^{*}$ and $\tilde{Q}=I-Q: H \rightarrow \operatorname{Im} L$. Let $L_{0}$ stand for the restriction of $L$ to $\operatorname{Im} L^{*} \cap$ $D(L)$. Hence $L_{0}$ is injective and by the assumptions, its inverse $K=L_{0}^{-1}: \operatorname{Im} L \rightarrow \operatorname{Im} L^{*} \cap$ $D(L)$ is bounded. Let $N: H \rightarrow H$ be a given mapping and $h \in H$. We consider the semilinear equation

$$
L u-N(u)=h, \quad u \in D(L)
$$

which can be reformulated as follows (see [2]).

Lemma 7.1. With $L, P, \tilde{P}, Q, \tilde{Q}$ as above, let $\mathscr{F}: H \rightarrow H$ be a linear homeomorphism such that

$$
\mathscr{L}(\operatorname{Ker} L)=\operatorname{Ker} L^{*}
$$

Then $u \in D(L)$ satisfies the equation

$$
L u-N(u)=h
$$

if and only if

$$
\tilde{P}(u-K \tilde{Q} N(u))+P \mathscr{F}^{*} N(u)=\hat{h},
$$

where $\hat{h}=K \tilde{Q} h-P \mathscr{F}^{*} h$.

Define $F: H \rightarrow H$ by

$$
F(u)=\tilde{P}(u-K \tilde{Q} N(u))+P \mathscr{F}^{*} N(u), \quad u \in H .
$$

Assume that $K$ is compact and $N: H \rightarrow H$ is bounded, demicontinuous and of class $\left(S_{+}\right)_{F}$. Then $\mathscr{F}^{*} N \in\left(S_{+}\right)_{P}$ and $F \in \mathscr{F}$, that is, the degree

$$
\mathrm{d}(F, G, \hat{h})=\mathrm{d}\left(\tilde{P}(I-K \tilde{Q} N)+P \mathscr{F}^{*} N, G, K \tilde{Q} h-P \mathscr{F}^{*} h\right)
$$

is well-defined for any open bounded set $G \subset H$ such that $h \notin(L-N)(\partial G \cap D(L))$. Hence the degree theory constructed in this note is applicable to solve (7.3) with a nonsymmetric linear operator $L$. In view of applications it is useful to recognize the case where $F=I$, the normalizing map for which the degree is +1 , when $\hat{h} \in G$.

Lemma 7.2. Let $\operatorname{Ker} L^{*}=\mathscr{F}(\operatorname{Ker} L)$ and $N_{0}=Q\left(\mathscr{F}^{*}\right)^{-1}$. Then $N_{0}$ is bounded, continuous and of class $\left(S_{+}\right)_{q}$. Moreover,

$$
F=\tilde{P}\left(I-K \tilde{Q} N_{0}\right)+P \mathscr{F}^{*} N_{0}=I .
$$

Proof. To prove the first assertion assume that

$$
u_{n} \rightarrow u, \quad \tilde{P} u_{n} \longrightarrow \tilde{P} u, \quad \limsup _{n \rightarrow \infty}\left\langle N_{0}\left(u_{n}\right), \mathscr{E} P\left(u_{n}-u\right)\right\rangle \leq 0 .
$$


Then,

$$
\begin{aligned}
\limsup \left\langle N_{0}\left(u_{n}\right), \mathscr{E} P\left(u_{n}-u\right)\right\rangle & =\limsup \left\langle Q\left(\mathscr{F}^{*}\right)^{-1}\left(P u_{n}\right), \mathscr{E}\left(P u_{n}-P u\right)\right\rangle \\
& =\limsup \left\langle\left(\mathscr{E}^{*}\right)^{-1}\left(P u_{n}\right), \mathscr{E}\left(P u_{n}-P u\right)\right\rangle \\
& =\limsup \left\|P u_{n}-P u\right\|^{2} \leq 0
\end{aligned}
$$

implying $u_{n} \rightarrow u$ and hence $N_{0}$ is bounded, continuous and of class $\left(S_{+}\right)_{\mathscr{q}}$. A direct calculation gives

$$
F=\tilde{P}\left(I-K \tilde{Q} Q\left(\mathscr{F}^{*}\right)^{-1}\right)+P \mathscr{F}^{*} Q\left(\mathscr{F}^{*}\right)^{-1}=\tilde{P}+P \mathscr{F}^{*} Q\left(\mathscr{F}^{*}\right)^{-1},
$$

where

$$
P \mathscr{F}^{*} Q\left(\mathscr{E}^{*}\right)^{-1}=P \mathscr{F}^{*}(I-\tilde{Q})\left(\mathscr{F}^{*}\right)^{-1}=P-P \mathscr{E}^{*} \tilde{Q}\left(\mathscr{F}^{*}\right)^{-1}=P
$$

due to the fact that $\mathscr{F}^{*}(\operatorname{Im} L)=\operatorname{Im} L^{*}$ and thus $P \mathscr{F}^{*} \tilde{Q}\left(\mathscr{F}^{*}\right)^{-1}=0$. Consequently, $F=I$ and the proof is complete.

For further applications to problems involving non-symmetric linear part, we refer to $[2]$.

\section{References}

[1] J. Berkovits, On the Leray-Schauder formula and bifurcation, J. Differential Equations 173 (2001), no. 2, 451-469.

[2] J. Berkovits and C. Fabry, Semilinear problems with a non-symmetric linear part having an infinite dimensional kernel, Port. Math. (N.S.) 61 (2004), no. 4, 439-459.

[3] J. Berkovits and V. Mustonen, An extension of Leray-Schauder degree and applications to nonlinear wave equations, Differential Integral Equations 3 (1990), no. 5, 945-963.

[4] J. Berkovits and M. Tienari, Topological degree theory for some classes of multis with applications to hyperbolic and elliptic problems involving discontinuous nonlinearities, Dynam. Systems Appl. 5 (1996), no. 1, 1-18.

[5] H. Brezis, Équations et inéquations non linéaires dans les espaces vectoriels en dualité, Ann. Inst. Fourier (Grenoble) 18 (1968), no. 1, 115-175 (French).

[6] H. Brezis and L. Nirenberg, Characterizations of the ranges of some nonlinear operators and applications to boundary value problems, Ann. Scuola Norm. Sup. Pisa Cl. Sci. (4) 5 (1978), no. 2, 225-326.

[7] F. E. Browder, Fixed Point Theory and Nonlinear Problems, Proc. Sympos. Pure Math., vol. 39, Part 2, American Mathematical Society, Rhode Island, 1983.

[8] A. Fonda and J. Mawhin, Iterative and variational methods for the solvability of some semilinear equations in Hilbert spaces, J. Differential Equations 98 (1992), no. 2, 355-375.

[9] J. Mawhin, Equivalence theorems for nonlinear operator equations and coincidence degree theory for some mappings in locally convex topological vector spaces, J. Differential Equations 12 (1972), 610-636.

[10] _ Topological Degree Methods in Nonlinear Boundary Value Problems, CBMS Regional Conference Series in Mathematics, vol. 40, American Mathematical Society, Rhode Island, 1979. 
[11] Nonlinear functional analysis and periodic solutions of semilinear wave equations, Nonlinear Phenomena in Mathematical Sciences (Arlington, Tex, 1980), Academic Press, New York, 1982, pp. 671-681.

[12] J. Mawhin and M. Willem, Perturbations nonlinéaires d'opérateurs linéaires à noyau de dimension infinie, C. R. Acad. Sci. Paris Sér. A-B 287 (1978), no. 5, A319-A322 (French).

[13] _ Range of nonlinear perturbations of linear operators with an infinite-dimensional kernel, Ordinary and Partial Differential Equations (Proc. 5th Conf., Univ. Dundee, Dundee, 1978), Lecture Notes in Math., vol. 827, Springer-Verlag, Berlin, 1980, pp. 165-181.

[14] Operators of monotone type and alternative problems with infinite-dimensional kernel, Recent Advances in Differential Equations (Trieste, 1978), Academic Press, New York, 1981, pp. 295-307.

J. Berkovits: Department of Mathematical Sciences, University of Oulu, P.O. Box 3000, 90014 Oulu, Finland

E-mail address: juha.berkovits@oulu.fi

C. Fabry: Institut de Mathématique Pure et Appliquée, Université Catholique de Louvain, Chemin du Cyclotron 2, 1348 Louvain-la-Neuve, Belgium

E-mail address: fabry@math.ucl.ac.be 


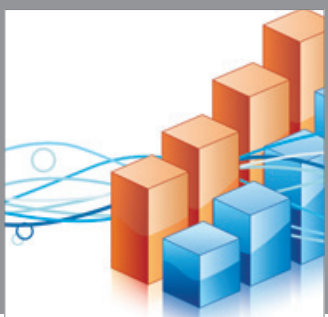

Advances in

Operations Research

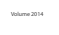

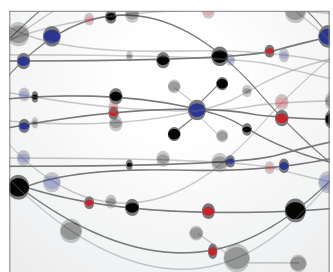

\section{The Scientific} World Journal
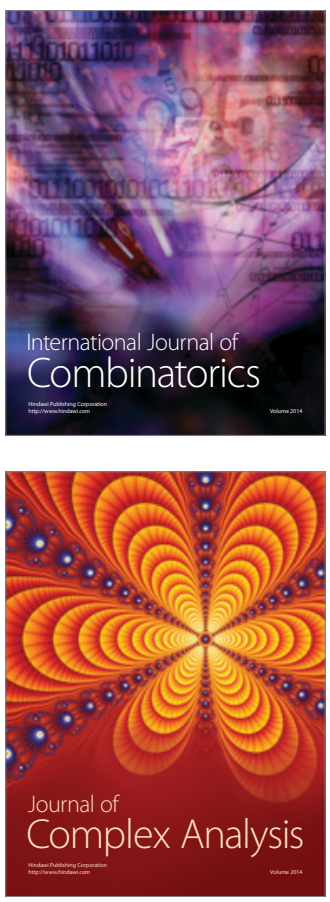

International Journal of

Mathematics and

Mathematical

Sciences
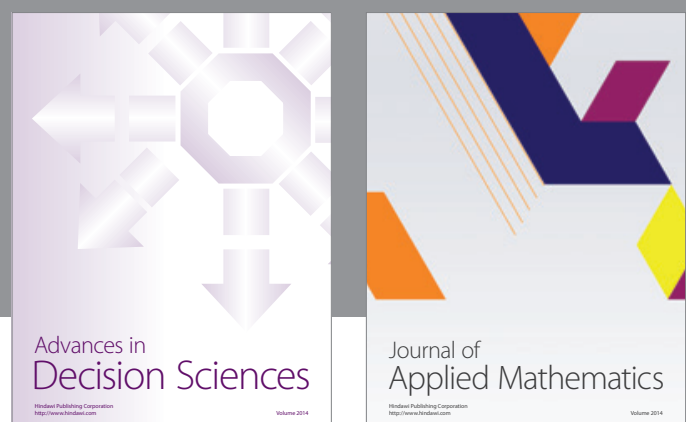

Journal of

Applied Mathematics
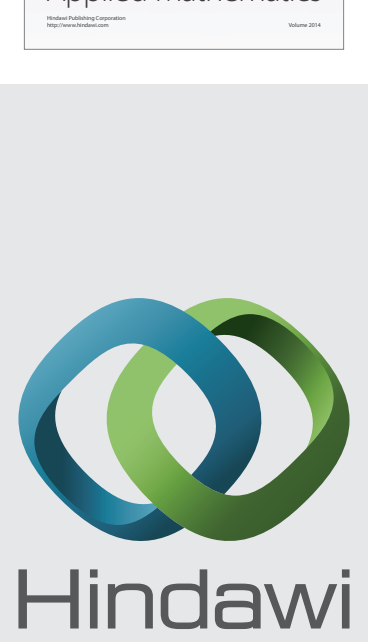

Submit your manuscripts at http://www.hindawi.com
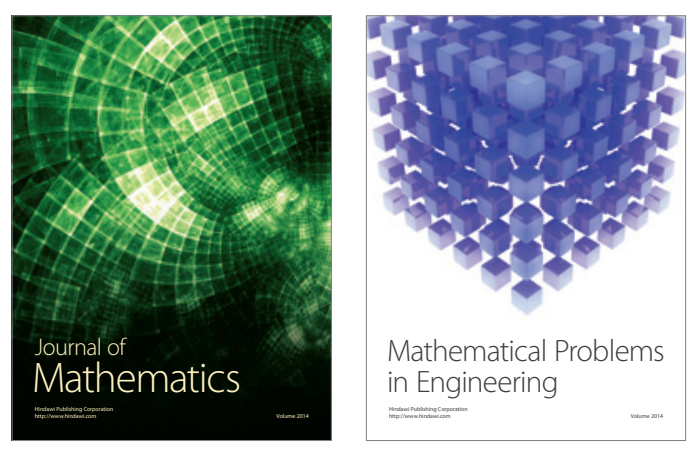

Mathematical Problems in Engineering
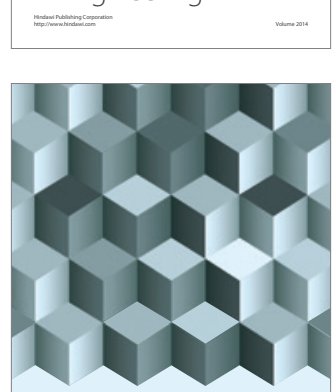

Journal of

Function Spaces
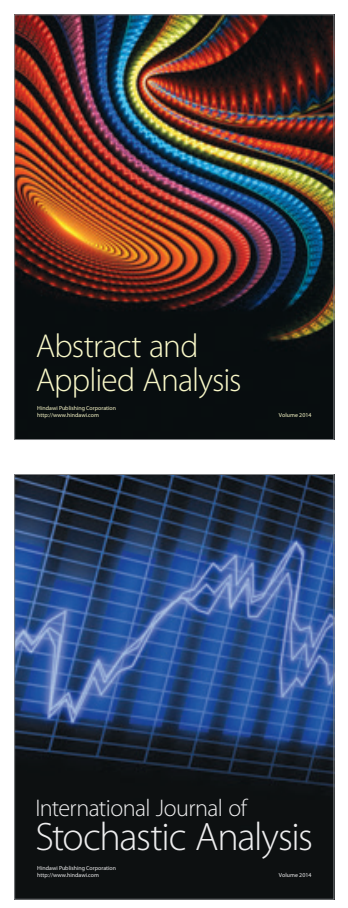

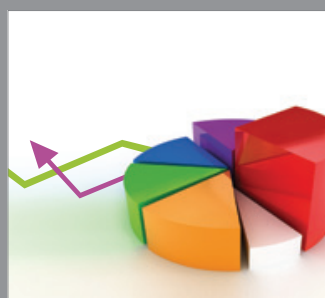

ournal of

Probability and Statistics

Promensencen
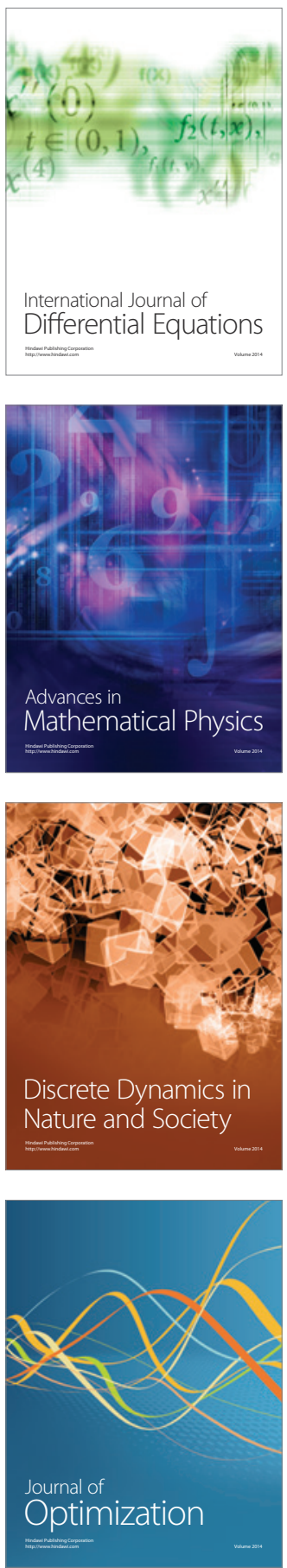